\title{
Improving Patient Safety: Lessons from Other Disciplines
}

\section{KAREN GOLEMBOSKI}

\section{LEARNING OBJECIVES}

1. Compare data collection methods used to describe error incidence in healthcare and the aviation industry.

2. Discuss regulatory requirements and public accessibility of information regarding errors for aviation and medicine.

3. State the benefits of checklists in aviation and medicine.

4. Describe the improvements in healthcare quality as a result of implementation of electronic health record systems.

5. Discuss the applications and benefits of team-based training in healthcare.

ABBREVIATIONS: AHRQ, Agency for Research and Quality; CAP, College of American Pathologists, CPOE, computerized provider order entry; CRM , crew resource management; FAA, Federal Aviation Administration; FDA, Food and Drug Administration; HAI, healthcare associated infections; LOSA, line operations safety audit; NTSP, National Transportation Safety Board; PSO, Patient Safety Organization; SRE, serious reportable event

INDEX TERMS: Quality Improvement; Healthcare Quality Assurance

Clin Lab Sci 2011;24(2):114

Karen Golemboski, Ph.D., MT(ASCP), Bellarmine University, Medical Laboratory Science Department, Louisville KY 40205

Address for Correspondence: Karen Golemboski, Ph.D., MT(ASCP), Bellarmine University, Medical Laboratory Science Department, 2001 Newburg Rd., Louisville KY 40205, (502)272-8387, kgolemboski@bellarmine.edu
Quality of clinical laboratory services means more than providing analytically accurate test information. The goal is to improve patient outcomes without imposing harm. ${ }^{1,2}$ Quality improvement in laboratory services requires focusing on both analytical excellence and patient-centered aspects of care. Technology in the clinical laboratory is complex and changes rapidly, factors which may increase the opportunity for errors to occur. Intentional system design and analysis can help reduce errors and mitigate the effects of those that occur. ${ }^{3}$ Many industries have made quality and safety improvement a priority for their services and products. Laboratorians can learn from other industries' experiences, selecting approaches likely to result in improved quality and safety of laboratory services.

\section{Aviation Industry}

One model for improving healthcare is the safety improvement experience of the aviation industry. Although the characteristics and consequences of error in aviation are different than those in medicine (i.e., few highly visible incidents, each involving many lives, versus many individual incidents which may not be reported or even acknowledged), the two fields have common attributes. Both involve complex technology and highly trained specialists who share some aspects of professional culture. ${ }^{4}$ Certain principles of error management, as practiced by aviation, may be applicable to medical laboratory science.

A necessary starting point for quality improvement in any system is the collection and evaluation of information regarding the frequency and nature of incidents. Most errors that occur in a professional setting are classified as "blameless" and indicate the need to modify systems, as opposed to disciplining 


\section{FOCUS: PATIENT SAFETY AND THE MEDICAL LABORATORY}

individuals. To understand the corrective action needed, it is essential to know what errors occur. ${ }^{5}$

The aviation industry developed consistent methods to collect data on error incidence and to disseminate information regarding safety in response to public and regulatory concerns. Aviation error data are collected from voluntary sources (confidential surveys and nonpunitive reporting systems) and from expert observers, the line operations safety audit (LOSA). Significant incidents must be reported to the Federal Aviation Administration (FAA) or the National Transportation Safety Board (NTSB). Public access to aviation safety information is available through the NTSB and FAA. ${ }^{4}$ Errors detected by these systems are classified into five primary categories: violation, or non-compliance with standards; procedural errors; communications failure; proficiency errors, due to lack of information or technical skill; and decision error, in which a choice is made that increases the risk of an error. Most errors are non-compliance incidents, although those with the highest risk of adverse consequence are errors of proficiency or decision. Significant events are usually not the result of a single mistake, but rather represent the accumulation of a number of contributing events. ${ }^{4}$

\section{Error Reporting Programs}

Compared with aviation safety information, reporting of medical quality is less well established. Limited information regarding medical errors is collected in the United States primarily due to concerns about legal liability. ${ }^{6}$ Mandatory error-reporting systems are limited to serious incidents, although the aviation industry has demonstrated that valuable information can be gleaned from reporting of less-serious occurrences. Twentyseven states and the District of Columbia have medical error reporting systems, many administered by the Agency for Healthcare Research and Quality (AHRQ). Most of the state regulations require reports for some or all of the items on the National Quality Forum list of 28 Serious Reportable Events (SREs), which includes wrong-site surgeries, adverse effects related to use of a product or device, patient protection events, environmental events, criminal events, and care management events that result in serious disability or death. Twelve states currently require reporting of healthcare-associated infections (HAI); in Florida, hospital-specific HAI information is available. Although many state registries are hampered by lack of funding, and reporting systems are variable in format and extent, ${ }^{7}$ significant advances in healthcare quality have resulted. In Indiana, an emphasis on elimination of pressure sores decreased incidence by $30 \%$ over 15 months. ${ }^{8}$ Pennsylvania's Patient Safety Authority reported a 5\% decrease in reports of post-surgical infection from 2005 to 2007, and introduced standardized color-coding of patient wristbands after an employee who worked at two hospitals used the wrong color wristband and mistakenly designated a patient as "Do Not Resuscitate". ${ }^{8}$

Voluntary systems, such as the Medication Errors Reporting Program of the United States Pharmacopoeia, encourage confidential reporting of near misses in addition to serious events. These reports often provide valuable information that can suggest system modifications to reduce subsequent errors. ${ }^{9}$ To promote voluntary reporting, AHRQ created the Patient Safety Organization (PSO) program in 2009, describing requirements to establish entities authorized to collect and facilitate analyses of information about errors reported by healthcare organizations. One goal of the PSO program is to develop a digital aggregated database in a common format in order to analyze information and identify improvements in patient outcomes. Information gathered by PSOs is confidential and inaccessible to legal discovery. ${ }^{10}$ PSOs are responsible for collecting and analyzing patient safety data, providing feedback to facilities, and assisting with implementation of improvements. Organizations currently approved as PSOs include not-for-profit and for-profit entities; insurers and regulatory agencies do not qualify to serve as a PSO. The program is not federally-funded, and cost may be a hurdle for participation, although some states now require hospitals to contract with a PSO as evidence of quality improvement efforts. ${ }^{11}$

The public has access to certain medical error data through PSOs and state agencies, some of which is facility-specific. ${ }^{11}$ However, the type of safety information sought by consumers is often different than that reported by healthcare facilities, such as evaluating the hospital experience from the perspective of the 


\section{FOCUS: PATIENT SAFETY AND THE MEDICAL LABORATORY}

patient, appropriate pain relief, respectful treatment, and clear instructions for post-discharge care. Evaluation of publicly reported safety data is further complicated by variability in data collection and analysis. As the use of PSOs and other reporting mechanisms become more readily available, data collection will become standardized and quality benchmarks will be established, allowing both consumers and healthcare professionals to make valid comparisons and to set clear goals for improvement. ${ }^{12}$

Information concerning errors in laboratory medicine is no more comprehensive nor consistent than other medical errors. Although laboratory proficiency testing and quality control processes assess accuracy and precision of analytical test results, no standard mechanism exists to collect data about errors occurring in the pre-analytic, analytic and post-analytic phases of laboratory testing. Studies have indicated that more errors occur in the pre-analytical phase (46-68\%) than the analytic phase (7-13\%). ${ }^{13}$ Patient misidentification and unacceptable specimen quality due to hemolysis, clots, insufficient quantity, inappropriate container as well as improper test selection are common errors identified in the pre-analytic phase. ${ }^{14}$ Post-analytical problems include breakdowns in the transmission of results and follow-up required as indicated by test results. Gaps, breaches in continuity of care which lead to errors, are often detected at the interface of more than one area of responsibility or professional role, which emphasizes the need for attention to all aspects of patient testing. ${ }^{15,}{ }^{16}$ Developing standardized criteria for data collection and quality improvement methods to examine the entire testing process is important for participation in public reporting systems and improving laboratory services. ${ }^{17}$

\section{Methods and Technology to Standardize Healthcare Processes}

Anesthesiology was the first medical specialty to effectively address patient safety, using a combination of techniques first validated in other industries. Standardization of procedures, analysis of errors (including closed malpractice claims), application of technological solutions such as engineered safety devices which physically avert errors (e.g., standardized gas connectors which prevent incorrect installation), and development of evidence-based practice guidelines have contributed to decreasing anesthesia-related deaths from 2 of 10,000 patients to 1 in 200,000-300,000 patients. ${ }^{18,19}$ In 1986, the American Society of Anesthesiology and the Food and Drug Administration (FDA) proposed a checklist to standardize preanesthesia care. Similar guidelines were later adopted internationally. ${ }^{20}$

Use of checklists standardizes procedures in aviation and processes of healthcare. Checklists were introduced in aviation prior to World War II. As aircraft became more complex, the number of routine tasks to be completed before and during flight exceeded what could realistically be remembered by every pilot. The use of checklists, with simple reminders of each step to complete, led to safe operation of the most advanced airplanes by ensuring that steps and checkpoints were not omitted, either inadvertently or because they seemed unimportant. ${ }^{21}$ Similar procedures are used in manufacturing, food inspection, and pharmaceutical clinical trials. Although healthcare involves situations which are often less predictable than operating airplanes, the introduction of medical checklists in the last decade suggests that decreasing variability may improve healthcare outcomes, as well. A simple 5-step checklist implemented at Johns Hopkins decreased the incidence of infections associated with central line insertion from $11 \%$ to zero over the course of one year. ${ }^{22}$ The Pronovost checklist includes the following five steps for central line insertion: wash hands, disinfect the patient's skin, cover the patient with sterile drapes, wear mask/hat/gown/gloves, and apply a sterile dressing after line insertion. By standardizing each step in a process with evidence-based procedures, and measuring the outcomes of their use, checklists have consistently produced improvements in patient outcomes, including end-of-life care and prediction of successful ventilator weaning. ${ }^{23}$

Checklist use improves the system, as well as patients' outcome. Implementation of a checklist to improve care for patients on ventilators improved adherence to evidence-based practice by $66 \%{ }^{24}$ The central line care bundle, a set of best practices, includes the hand hygiene and barrier protection elements of the Pronovost checklist and adds site selection (i.e., 


\section{FOCUS: PATIENT SAFETY AND THE MEDICAL LABORATORY}

avoidance of femoral line insertion) and daily review of the need for continued use of a central line. Use of the central line care bundle in a critical care unit over a 2 year period reduced infection rates from 5.0 to 0.90 per 1000 catheter days. ${ }^{25}$ An infection control care bundle targeting Clostridium difficile decreased infections by $78 \% .{ }^{26}$ Despite these dramatic results, checklists are not yet ubiquitous in medicine. Successful implementation of checklists requires a comprehensive effort that includes a summary of tasks and data collection along with subsequent analysis and feedback, within a culture of requiring performance standards. ${ }^{27}$

Checklists are not unfamiliar to laboratory professionals. The value of the standard operating procedure is well-established in the clinical laboratory and is endorsed by laboratory accrediting organizations such as the College of American Pathology (CAP) and The Joint Commission. The laboratory may be ahead of the safety curve in this respect; however, it also means that this type of intervention may have limited potential for additional quality improvement in the laboratory compared to other areas of healthcare. The laboratory's best opportunity for improvement using checklists is to address errors occurring in the pre- and post-analytical phases of the testing process.

Implementation of electronic health records (EHR) provides mechanisms with the potential to improve patient outcomes, including increased access to measures of quality and standardization of communication among areas of service. One feature of EHR, computerized provider order entry (CPOE), decreased medication errors by $55-83 \%$ in multiple studies. ${ }^{28}$ Computerized orders eliminate errors resulting from illegible handwriting and transcription errors. EHRs may also incorporate clinical pathway standards with recommendations for medications or other interventions, which may minimize treatment errors. $^{29}$ Use of CPOE favorably impacts both timeliness and accuracy of laboratory orders; turnaround time for tests in the intensive care unit decreased by $50 \%$ in one study ${ }^{30}$ and transfusion complications decreased from $6.1 \%$ to $2.7 \%$ in another. ${ }^{31}$ The use of pre-determined test panels (order sets), monitoring alerts and enhanced interpretation of results may increase efficacy of laboratory testing. ${ }^{32}$

\section{Communication and Teamwork}

Professionals involved in high-risk, high-complexity disciplines share interpersonal characteristics that may impact both safe performance and function as team members. Both pilots and healthcare professionals tend to underestimate their own commission of errors. ${ }^{4}$ Physicians are even less likely than pilots to acknowledge the effects of fatigue on quality of performance, and surgeons are more likely to perceive their behavior as "teamwork" than nurses or other physicians present in the operating room. ${ }^{33}$ Nontechnical skills such as communication, decisionmaking, and teamwork are often implicated in medical errors $^{34}$ and poor communication may have contributed to $70 \%$ of sentinel events reported to The Joint Commission in 2005. ${ }^{35}$ Medical education values autonomy and communication in medicine tends to follow a hierarchical structure that inhibits teamwork. This is complicated further by training and socializing all healthcare professionals as individuals yet expecting each to perform as team members. All of these characteristics contribute to conflict among healthcare professionals, which has been associated with reporting of significant medical errors. ${ }^{36}$ Recognition of the importance of communication in quality patient care has led the Institute of Medicine to call for an increased focus on inter-professional education. ${ }^{2}$

Crew resource management (CRM) training, a teambased learning method used by the aviation industry, addresses non-technical performance issues that often contribute to errors. Components of CRM include recognition of roles and responsibilities of team members, communication methods, ongoing assessment and review of the situation, anticipation of need, and procurement of necessary resources. Team members learn to recognize cognitive errors (situation awareness) and conflict resolution. Although aviation errors occur infrequently and therefore there are no published data on decreased errors after CRM training, outcomes include behavioral improvements such as improved teamwork and increased awareness of error potential. ${ }^{37}$ Similar programs are used in interdisciplinary training models in medicine, although the " $\mathrm{C}$ " usually represents Crisis in medical settings for scenarios in the emergencydepartment, resuscitation units, or labor and delivery. 


\section{FOCUS: PATIENT SAFETY AND THE MEDICAL LABORATORY}

In both aviation and medicine, CRM training often employs simulators. Simulation-based training provides the obvious benefit of practice without endangering patients. Review and debriefing provides feedback often not available from real-world incidents. Participation in CRM exercises has increased self-reporting of errors across healthcare professions, awareness of potential errors, and improved teamwork behavior. ${ }^{33,37}$ A key benefit of simulation-based exercises is information about latent threats, identified as system characteristics that may not individually create an incident, but may increase the chance of serious error when combined with other circumstances. ${ }^{4}$ Crisis situations that end badly are often subject to review using quality improvement methods such as root cause analysis; however, many errors occur during the course of routine healthcare delivery and involve individuals who do not routinely participate in crisis management. Extending team-based training to non-crisis situations is a valid method to address errors that occur in routine healthcare processes. Aviation's LOSA procedure has been expanded to include safety audits of on-the ground operations. In healthcare as well, non-technical, teambuilding skills should be addressed with a variety of teams and situations in order to maximize leadership development and effective communication skills without minimizing complexity of inter-professional care. $^{38}$

Interdisciplinary simulator-based education usually includes physicians, nurses, respiratory therapists, or emergency responders (or students in these fields). Available scenarios provided with simulators do not generally include active participation by laboratory professionals. Although laboratory test results are often an important part of the situation portrayed, the information is provided to participants by the facilitator. To gain the benefits of participation in teambased interdisciplinary instruction, laboratory scientists may need to intentionally request inclusion, or develop specific scenarios which involve interaction between laboratory and non-laboratory personnel.

Evidence from other industries suggests that a dedication to quality requires commitment from leadership and attention to cultural, as well as technical factors. ${ }^{39}$ Incorporating the facility's commitment to quality patient care into the laboratory's mission may encourage a broader, non-traditional conception of quality which embraces not only analytical proficiency and turnaround time, but care which is also equitable, effective, efficient, and patient-centered.

\section{Summary}

Other industries and certain healthcare specialties have employed a variety of methods to improve safety and quality of services. Techniques such as industry-wide standardized collection and reporting of error data, standardization of practice through checklists, application of electronic health records, and simulatorbased interdisciplinary training have improved outcomes in aviation, anesthesiology, and surgery. Although traditionally the clinical laboratory has concentrated on analytical performance, pre- and postanalytical aspects of laboratory services may also be improved through the application of these methods.

\section{REFERENCES}

1. Kohn L, Corrigan J, Donaldson M. eds. To err is human: building a safer health system. Washington DC: Institute of Medicine, Committee on Quality of Health Care in America. 2000; $155-97$.

2. Institute of Medicine. Crossing the Quality Chasm: A new health system for the $21^{\text {st }}$ century. Washington, DC: National Academic Press; 2001;1-22.

3. Nolan TW. System changes to improve patient safety. BMJ. 2000;320:771-3.

4. Helmreich RL. On error management: lessons from aviation. BMJ, 2000;320:781-5.

5. Reason J. Safety in the operating theatre, Part 2: Human error and organizational failure. Qual Saf Health Care. 2005;14:5661.

6. Wu AJ. The Second Victim: The doctor who makes the mistake needs help too. BMJ. 2000;320:726-7.

7. A national survey of medical error reporting laws. Yale J Health Policy Law Ethics. 2009;9 (1):201-86.

8. The Power of Safety: State Reporting Provides Lessons in Reducing Harm, Improving Care. National Quality Forum, June 2010;12

9. Cohen MR. Why error reporting systems should be voluntary. BMJ. 2000;320:728-9.

10. Montoya ID. Patient safety and quality improvement: a policy assessment. Clin Lab Sci. 2010;23(4):212-8.

11. DerGurahian J. Still hard to share. Modern Healthcare. 2009;39(41):30-2.

12. Monroe A. Consumer involvement - a vital piece of the quality quilt. Qual Saf Health Care. 2002;11:181-5.

13. Plebani M. Errors in clinical laboratories or errors in laboratory medicine? Clin Chem Lab Med. 2006;44(6):750-9. 


\section{FOCUS: PATIENT SAFETY AND THE MEDICAL LABORATORY}

14. Lippi G, Fostini R, Guidi G. Quality improvement in laboratory medicine: extra-analytical issues. Clin Lab Med. 2008;28(2):285-94.

15. Cook RI, Render M, Woods DD. Gaps in the continuity of care and progress on patient safety. BMJ. 2000;320:791-4.

16. Carraro P, Plebani M. Errors in a stat laboratory: types and frequencies 10 years later. Clin Chem. 2007;53(7) 1338-42.

17. Shahangian S, Snyder SR. Laboratory medicine quality indicators: A review of the literature. Am J Clin Pathol. 2009;131(3):418-31.

18. Gaba DM. Anaesthesiology as a model for patient safety in health care. BMJ. 2000;320:785-8.

19. Lanier WL. A three-decade perspective on anesthesia safety. American Surgeon. 2006;72(11):985-9.

20. Auerbach AD, Murff HJ, Islam SD. Pre-anesthesia checklists to improve patient safety. In: Making Health Care Safer: A Critical Analysis of Patient Safety Practices. Evidence Report/Technology Assessment: Number 43.-Markowitz AJ, Shojania KG, Duncan BW, McDonald KM, Wachter RM, eds. Rockville, MD: Agency for Healthcare Research and Quality. 2001. Chapter 23;265-70.

21. Gawande A. The Checklist Manifesto. New York: Henry Holt and Company; 2009;32-7.

22. Berenholtz SM, Pronovost PJ, Lipsett PA, Hobson D, Earsing K,Farley JE, et al. Eliminating catheter-related bloodstream infections in the intensive care unit. Crit Care Med 2004;32(10):2014-20.

23. Hales BM, Pronovost PJ. The checklist - a tool for error management and performance improvement. J Crit Care. 2006;21:231-5.

24. Berenholtz SM, Milanovich S, Faircloth A, Prow DT, Earsing $\mathrm{K}$, Lipsett $\mathrm{P}$, et al. Improving care for the ventilated patient. Joint Commission Journal of Quality and Safety. 2004;30(4):195-204.

25. Galpern D, Guerrero A, Tu A, Fahoum B. Effectiveness of a central line bundle campaign on line-associated infections in the intensive care unit. Surgery. 2008;144:492-5.

26. Muto CA, Blank MK, Marsh JW, Vergis EN, O'Leary MM, Shutt KA, et al. Control of an outbreak of infection with the hypervirulent Clostridium difficile BI strain in a university hospital using a comprehensive "bundle" approach. Clin Inf Dis. 2007; 45:1266-73.

27. Bosk CL, Dixon-Woods M, Goeschel CA, Pronovost PJ. Reality check for checklists. Lancet. 2009;374(9688):444-5.
28. Bates DW. Using information technology to reduce rates of medication errors in hospitals.BMJ. 2000;320:788-91.

29. Malloch K. The electronic health record: an essential tool for advancing patient safety. Nursing Outlook. 2007;55(3):15961.

30. Thompson W, Dodek PM, Norena M, Dodek J. Computerized physician order entry of diagnostic tests in an intensive care unit is associated with improved timeliness of service. Crit Care Med. 2004;32(6):1306-9.

31. Rana R, Afessa B, Keegan M, Whalen F, Nuttail G, Evenson L, et al. Evidence-based red cell transfusion in the critically ill: quality improvement using computerized physician order entry. Crit Care Med. 2006;34(7):1892-7.

32. Westbrook JI, Georgiou A, Dimos A, Germanos T. Computerised pathology test order entry reduces laboratory turnaround times and influences tests ordered by hospital clinicians: a controlled before and after study. J Clin Pathol. 2006;59:533-6.

33. Sax HC, Browne P, Mayewski RJ, Panzer RJ, Hittner, et al. Can aviation-based team training elicit sustainable behavioral change? Arch Surg 2009;144 (12):1133-7.

34. Gawande A, Zinner M, Studdert, D, Brennan, T. Analysis of errors reported by surgeons at three teaching hospitals. Surgery. 2003;133:614-21.

35. Greenberg CC, Regenbogen SE, Studdert DM, Lipsitz SR, Rogers SO, et al. Patterns of communication breakdowns resulting in injury to surgical patients. J Am Coll Surg. 2007;204(4):533-40.

36. Baldwin DC, Daugherty SR. Interprofessional conflict and medical errors: results of a national multi-specialty survey of hospital residents in the US. Journal of Interprofessional Care. 2008;22(6):573-86.

37. Rudy SJ, Polomano R, Murray WB, Henry J, Marine R. Team management training using crisis resource management results in perceived benefits by healthcare workers. J Cont Ed Nursing. 2007;38(5):219-26.

38. Sutton G. Evaluating multidisciplinary health care teams: taking the crisis out of CRM. Australian Health Review. 2009;33(3):445-52.

39. Leape, L. Safe health care: are we up to it? BMJ.2000;320:7256. 